\title{
REACTIONS OF 4-HYDROXYCOUMARIN WITH HETEROCYCLIC ALDEHYDES
}

\author{
RENATA GAŠPAROVÁ ${ }^{1}$, KATARÍNA KOTLEBOVÁ ${ }^{1}$, \\ MARGITA LÁCOVÁ \\ ${ }^{1}$ Department of Chemistry, University of SS. Cyril and Methodius, J. Herdu 2, Trnava, \\ SK-917 01, Slovak Republic (gasparor@ucm.sk) \\ ${ }^{2}$ Department of Organic Chemistry, Comenius University, Mlynská dolina CH-2, \\ Bratislava, SK-842 15, Slovak Republic (lacova@fns.uniba.sk)
}

\begin{abstract}
Reactions of 4-hydroxycoumarin 1 with heterocyclic aldehydes 2-4 led to bis-4-hydroxycoumarin derivatives 5-7 under microwave irradiation as well as under the classical heating. The subsequent reactions of products 5-7 are described. 4,4'-Epoxydicoumarins 8, 9 were prepared by the reaction of 5-7 in acetic acid / p-toluenesulfonic acid medium. Compound $\mathbf{1 0}$ was prepared by the reaction of $\mathbf{5}$ in acetic anhydride in the presence sodium acetate. Dioxocine-1,15-dione $\mathbf{1 1}$ was prepared by the reaction of $\mathbf{6}$ with dichloromethane in sodium hydroxide-toluene.
\end{abstract}

Key words: coumarin, 4-oxo-4H-chromene, furan, furo[3,2-b]pyrrole, microwave

\section{Introduction}

Coumarins are biologicaly active compounds with antifungal, antineoplastic, antibacterial, spasmolytic or cytotoxic activity (STANCHEV et al., 2007; JUNG et al., 2004). 4-Hydroxycoumarin $\mathbf{1}$ and its derivatives, e.g. warfarin and dicoumarol are known as anticoagulants.

4-Hydroxycoumarin 1 reacts with aromatic or aliphatic aldehydes to give bis-4hydroxycoumarin derivatives (GIOVANI et al., 1991; MANOLOV et al., 2006; MAO et al., 2002), which are interesting for their biological activity and they can serve as intermediates for synthesis of various heterocycles (HAMDI et al., 2008; YAMASHITA et al., 1987).

\section{Experimental}

Melting points of products were determined on a Kofler hot plate apparatus and are uncorrected. All solvents were predistilled and dried appropriately prior to use. ${ }^{1} \mathrm{H}$ NMR spectra were obtained on a $300 \mathrm{MHz}$ spectrometer VARIAN GEMINI 2000 in $\mathrm{CDCl}_{3}$ or DMSO- $\mathrm{d}_{6}$ or $\mathrm{CF}_{3} \mathrm{COOD}$ with tetramethylsilane as an internal standard.

All microwave experiments were performed in a Panasonic NN-E205 type microwave oven. The apparatus was adapted for laboratory applications; n-hexane was used as coolant for the condenser.

\subsection{General procedure for 5 and 6}

A) Classical heating. A mixture of 4-hydroxycoumarin 1 (0.002 mol) and 6-R-4-oxo$4 \mathrm{H}$-chromen-3-carbaldehyde 2 (or 5-arylfuran-2-carbaldehyde 3 ) $(0.001 \mathrm{~mol}$ ) in acetic 
anhydride $\left(15 \mathrm{~cm}^{3}\right.$ ), in the presence of a catalytic amount of $\mathrm{K}_{2} \mathrm{CO}_{3}$ (or AcOK) was heated at $80{ }^{\circ} \mathrm{C}$ for $3.5 \mathrm{~h}$. After cooling the solid product was filtered off and crystallized from ethanol.

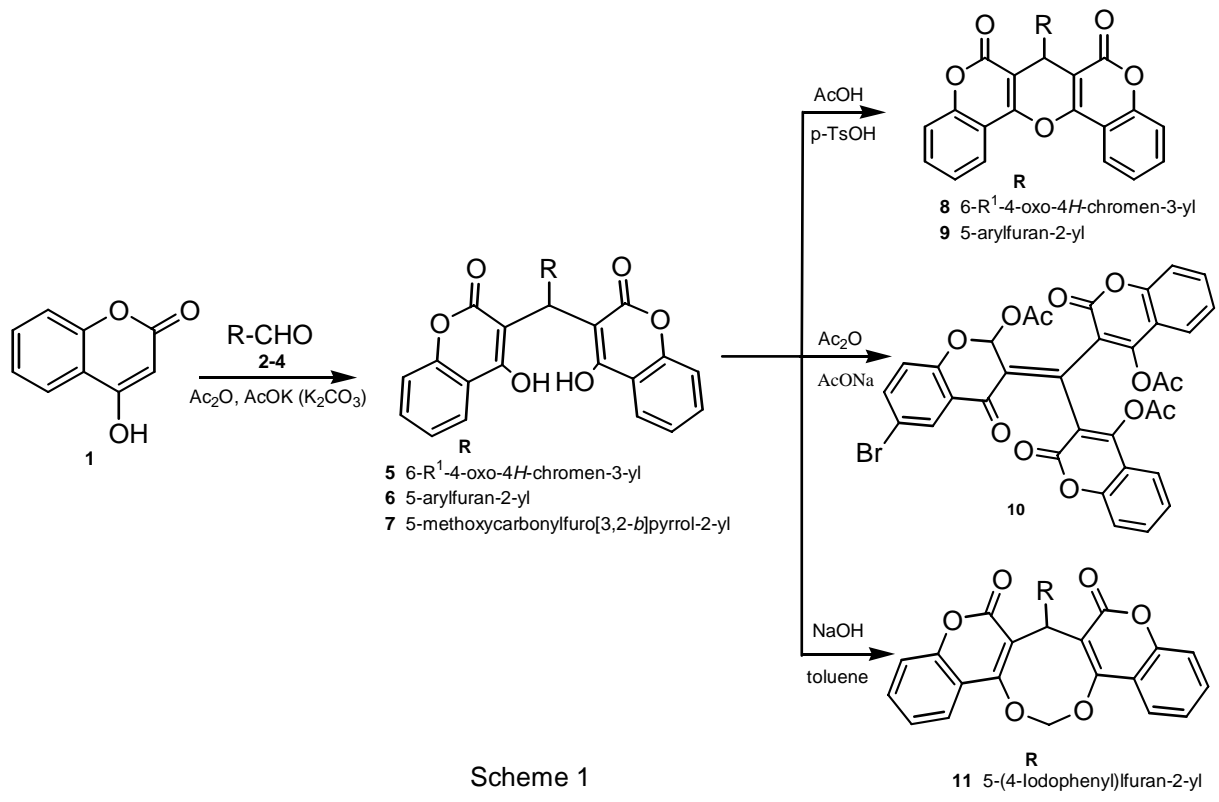

B) Microwave method. A mixture of 4-hydroxycoumarin 1 (0.002 mol) and 6-R-4oxo-4H-chromen-3-carbaldehyde 2 (or 5-arylfuran-2-carbaldehyde 3) $(0.001 \mathrm{~mol}$ ) in acetic anhydride $\left(4 \mathrm{~cm}^{3}\right)$, in the presence of a catalytic amount of $\mathrm{K}_{2} \mathrm{CO}_{3}$ (or AcOK) was irradiated in microwave oven for 4 min. The work-up was the same as described above.

2.1.1 3,3'-[(4-oxo-4H-chromen-3-yl)methylene]bis(4-hydroxy-2-oxo-2H-chromen-2one) $5 a$

For $\mathrm{C}_{28} \mathrm{H}_{16} \mathrm{O}_{8}$ (480.4): Mp: $275-280{ }^{\circ} \mathrm{C}$; react. time 3.5h (A), 4min (B); yield: $81 \%$ (A), 82\% (B); ${ }^{\mathrm{H}} \mathrm{NMR}\left(\mathrm{CDCl}_{3}\right)$ : 6.01 (d, $1 \mathrm{H}, \mathrm{CH} J=1.5 \mathrm{~Hz}$ ); 7.34-7.36 (m, $1 \mathrm{H}, \mathrm{H}-6$ ); 7.39 (dd, 4H, H-8', H-8", H-6', H-6"', ${ }^{3} J=7.97 \mathrm{~Hz}$ ); 7.46 (d, $1 \mathrm{H}, \mathrm{H}-8, \mathrm{~J}=8.25 \mathrm{~Hz}$ ); 7.59-7.61 (m, 2H, H-7', H-7"'); 7.67 (m, 1H, H-7); 7.92 (d, 1H, H-2, $J=1.7$ Hz); 8.04 (dd, 2H, H-5', H-5"', $J=7.9$ Hz); 8.11 (dd, 1H, H-5, $J=7.9$ Hz); 11.50 (bs, 2H, OH)

2.1.2 3,3'-[(6-Methyl-4-oxo-4H-chromen-3-yl)methylene]bis(4-hydroxy-2-oxo-2Hchromen-2-one) $5 \boldsymbol{b}$

For $\mathrm{C}_{29} \mathrm{H}_{19} \mathrm{O}_{8}$ (495.5): Mp: $276-278{ }^{\circ} \mathrm{C}$; react. time 3.5h (A); yield: $79 \%(\mathrm{~A}) ;{ }^{1} \mathrm{H}$ NMR (DMSO-d 6 ): 2.38 (s, 3H, CH $)$ ); 6.07 (d, $1 \mathrm{H}, \mathrm{CH}, J=1.5 \mathrm{~Hz}$ ); 7.19 (dd, $2 \mathrm{H}, \mathrm{H}-$ 8', H-8"', $J$ = 7.5, $1.1 \mathrm{~Hz}$ ); 7.24 (dd, 2H, H-6', H-6", $J$ = 7.5, 0.9 Hz); 7.46 (ddd, 2H, 
H-7', H-7"', $J=8.2,1.5 \mathrm{~Hz}$ ); 7.47-7.50 (m, 1H, H-7); 7.55 (dd, 1H, H-8, $J=8.6,1.6$ Hz); 7.69 (d, 1H, H-5, $J=1.5$ Hz); 7.78 (dd, 2H, H-5', H-5"', $J=7.9,1.5$ Hz); 7.83 (d, $1 \mathrm{H}, \mathrm{H}-2, J=1.5 \mathrm{~Hz}$ ); 17,2 (bs, 2H, OH)

2.1.3 3,3'-[(5-(4-Chlorophenyl)furan-2-yl)methylene]bis(4-hydroxy-2H-chromen-2one) $6 a$

For $\mathrm{C}_{29} \mathrm{H}_{17} \mathrm{ClO}_{7}$ (512.9): Mp: 265-269 ${ }^{\circ} \mathrm{C}$; react. time 93h (A), 1.5h (B); yield: 68\% (A), 70\% (B); ${ }^{1} \mathrm{H}$ NMR (DMSO- $d_{6}$ ): 5.15 (s, $\left.1 \mathrm{H}, \mathrm{CH}\right) ; 6.52$ (d, $1 \mathrm{H}, \mathrm{H}-4, J=3.3 \mathrm{~Hz}$ ); 6.91 (d, $1 \mathrm{H}, \mathrm{H}-3, J=3.3 \mathrm{~Hz}) ; 7.41$ (d, 2H, Ar - H, $J=8.5 \mathrm{~Hz}) ; 7.54-7.57$ (m, 6H, $\mathrm{Ar}-\mathrm{H}$ ); 7.77 (ddd, 2H, H - 7', $J=8.7,1.2 \mathrm{~Hz}$ ); 8.43 (dd, 2H, H - 5', $J=8.5,1.3 \mathrm{~Hz}$ ).

2.1.4 3,3'-[(5-(4-Nitrophenyl)furan-2-yl)methylene]bis(4-hydroxy-2H-chromen-2one) $6 \boldsymbol{b}$

For $\mathrm{C}_{29} \mathrm{H}_{17} \mathrm{NO}_{9}$ (457.5): Mp: $317-319{ }^{\circ} \mathrm{C}$; react. time $51 \mathrm{~h}$ (A), $1.5 \mathrm{~h}$ (B); yield: $60 \%$ (A), $62 \%$ (B); ${ }^{1} \mathrm{H}$ NMR (DMSO- $\left.d_{6}\right): 5.19$ (s, $\left.1 \mathrm{H}, \mathrm{CH}\right) ; 6.65$ (d, $1 \mathrm{H}, \mathrm{H}-4, J=3.3 \mathrm{~Hz}$ ); 7.23 (d, $1 \mathrm{H}, \mathrm{H}-3, J=3.3 \mathrm{~Hz}) ; 7.54-7.57$ (m, 8H, Ar - H); $7.82-7.84$ (m, 5H, Ar $\mathrm{H}) ; 8.24$ (d, 2H, H -8"', $J=8.3 \mathrm{~Hz}$ ).

2.1.5 3,3'-(\{5-[4-(3-Trifluoromethyl)phenyl]furan-2-yl\}methylene)bis(4-hydroxy-2Hchromen-2-one) $6 \mathrm{c}$

For $\mathrm{C}_{30} \mathrm{H}_{17} \mathrm{~F}_{3} \mathrm{O}_{7}$ (546.5): Mp: $257-260{ }^{\circ} \mathrm{C}$; react. time $70 \mathrm{~h}(\mathrm{~A}), 1.5 \mathrm{~h}$ (B); yield: $50 \%$ (A), 51\% (B); ${ }^{1} \mathrm{H}$ NMR (DMSO- $d_{6}$ ): 5.17 (s, $\left.1 \mathrm{H}, \mathrm{CH}\right) ; 6.65$ (d, $1 \mathrm{H}, \mathrm{H}-4, J=3.3 \mathrm{~Hz}$ ); 7.11 (d, $1 \mathrm{H}, \mathrm{H}-3, J=3.3 \mathrm{~Hz}) ; 7.58-7.61$ (m, 5H, Ar - H); $7.77-7.83$ (m, 5H, Ar $\mathrm{H}) ; 8.44$ (d, 2H, H -8"', $J=8.4 \mathrm{~Hz}$ ).

\subsubsection{3,3'-[(5-(4-Iodophenyl)furan-2-yl)methylene]bis(4-hydroxy-2H-chromen-2-one) 6d}

For $\mathrm{C}_{29} \mathrm{H}_{17} \mathrm{IO}_{7}$ (604.4): Mp: 248-252 ${ }^{\circ} \mathrm{C}$; react. time 70h (A), 1.5h (B); yield: $51 \%$ (A), $53 \%$ (B); ${ }^{1} \mathrm{H}$ NMR (DMSO- $d_{6}$ ): 5.14 (s, $\left.1 \mathrm{H}, \mathrm{CH}\right) ; 6.52$ (d, $1 \mathrm{H}, \mathrm{H}-4, J=3.3 \mathrm{~Hz}$ ); 6.92 (d, $1 \mathrm{H}, \mathrm{H}-3, J=3.3 \mathrm{~Hz}$ ); 7.35 (d, 2H, Ar - H, J = 8,7 Hz); $7.51-7.59$ (m, 4H, H-6',H-8'); 7.73 (d, 2H, Ar-H $J=8.7 \mathrm{~Hz}$ ); 7.83 (ddd, 2H, H - 7', $J=8.7,1.5 \mathrm{~Hz}$ ); 8.42 (dd, $2 \mathrm{H}, \mathrm{H}-5^{\prime}, \mathrm{J}=8.4,1.2 \mathrm{~Hz}$ ).

\subsection{Synthesis of Methyl 2-[bis(4-hydroxy-2H-chromen-3-yl)methyl]-4H- furo[3,2-b]pyrrole-5-carboxylate 7}

A) Classical heating. A mixture of 4-hydroxycoumarin 1 (0.002 mol) and methyl 2formyl- $4 H$-furo[3,2-b]pyrrole-5-carboxylate $4(0.001 \mathrm{~mol})$ in pyridine $\left(15 \mathrm{~cm}^{3}\right)$ was heated at $105{ }^{\circ} \mathrm{C}$ for $3 \mathrm{~h}$. After cooling the solid product was filtered off and crystallized from ethanol.

B) Microwave method. A mixture of 4-hydroxycoumarin 1 (0.002 mol) and 2-formyl$4 H$-furo[3,2-b]pyrrole-5-carboxylate $4(0.001 \mathrm{~mol})$ in pyridine $\left(4 \mathrm{~cm}^{3}\right)$ was irradiated in microwave oven for $3 \mathrm{~min}$. The work-up was the same as described above. 
For $\mathrm{C}_{27} \mathrm{H}_{15} \mathrm{O}_{9} \mathrm{~N}$ (497.4): Mp: $275-280{ }^{\circ} \mathrm{C}$; react. time $3 \mathrm{~h}(\mathrm{~A}), 3$ min (B); yield: $52 \%$ (A), 56\% (B); ${ }^{1} \mathrm{H}$ NMR (DMSO- $d_{6}$ ): 3.89 (s, 3H, $\mathrm{CH}_{3}$ ); 6.90 (s, $1 \mathrm{H}, \mathrm{CH}$ ); 7.33-7.40 (m, 4H, Ar-H); 7.71-7.79 (m, 2H, Ar-H); 7.97 (m, 2H, Ar-H); 8.28 (s, 1H, H-3); 8.66 (s, 1H, H-6); 9.05 (s, 1H, NH); 12.46 (d, 2H, 2 OH).

\subsection{General procedure for 8 and $\mathbf{9}$}

Compound 5 (or 6) $(0.2 \mathrm{mmol})$ in acetic acid $\left(5 \mathrm{~cm}^{3}\right)$ and catalytic amount of ptoluenesulfonic acid was heated at $80^{\circ} \mathrm{C}$. After cooling the solid was filtered off and crystallized in ethanol.

\subsubsection{3,3'-[(4-Oxo-4H-chromen-3-yl)methylene $]-4,4^{\prime}$-epoxydicoumarin 8}

For $\mathrm{C}_{28} \mathrm{H}_{14} \mathrm{O}_{7}$ (462.4): Mp: 330-335 ${ }^{\circ} \mathrm{C}$; react. time 2.5h; yield: 92\%; ${ }^{1} \mathrm{H}$ NMR (DMSO-d (D) 4.77 (s, 1H, CH; 7.41-7.42 (m, 1H, H-6); 7.51-7.78 (m, 4H, H-8', H-8" , H-6', H-6”); 7.66 (d, 1H, H-8, J = 8,4 Hz); 7.76-7.78 (m, 3H, H-7', H-7”); 7.88 (d, $1 \mathrm{H}, \mathrm{H}-5, J=7,8 \mathrm{~Hz}$ ); 8.45 (d, 2H, H-5', H-5'”, $J=7.8 \mathrm{~Hz}$ ); 8.71 (s, 1H, H-2).

\subsubsection{3,3'-[(5-(4-Nitrophenyl)furan-2-yl)methylene]-4,4'-epoxydicoumarin 9}

For $\mathrm{C}_{28} \mathrm{H}_{14} \mathrm{O}_{7}$ (439.4): Mp: 322-325 ${ }^{\circ} \mathrm{C}$; react. time 6h; yield: 62\%; ${ }^{1} \mathrm{H}$ NMR (DMSO-d D : 5.41 (s, $1 \mathrm{H}, \mathrm{CH}) ; 6.58$ (d, $1 \mathrm{H}, \mathrm{H}-4, J=3.4 \mathrm{~Hz}) ; 7.10$ (d, $1 \mathrm{H}, \mathrm{H}-3, J=$ $3.4 \mathrm{~Hz}$ ); 7.35 - 7.50 (m, 6H, Ar - H); 7.62 - 7.74 (m, 4H, Ar - H); $8.10-8.13$ (m, 2H, $\left.\mathrm{H}-3^{\prime}, 5^{\prime}\right)$.

\subsection{Synthesis of 2,4",4"'-triacethyl-3-[bis(4-hydroxy-2-oxo-2H- chromen-3-yl)methyl]-chromen-4-one 10}

A mixture of 4-hydroxycoumarin 1 (0.002 mol) and 6-bromo-4-oxo-4H-chromen3-carbaldehyde 2 in acetic anhydride $\left(5 \mathrm{~cm}^{3}\right)$ and catalytic amount of sodium acetate was heated at $80^{\circ} \mathrm{C}$ for $4.5 \mathrm{~h}$. After cooling the solid product was filtered off and crystallized from ethanol.

For $\mathrm{C}_{34} \mathrm{H}_{21} \mathrm{BrO}_{12}$ (701.4): Mp: 158-163 ${ }^{\circ} \mathrm{C}$; yield: 66\%; ${ }^{1} \mathrm{H}$ NMR $\left(\mathrm{CDCl}_{3}\right): 2.09$ (s, $\left.3 \mathrm{H}, \mathrm{CH}_{3}\right) ; 2$ x 2.18 (s, 3H, $\left.\mathrm{CH}_{3}\right) ; 7.12$ (d, $\left.1 \mathrm{H}, \mathrm{H}-8, J=8.5 \mathrm{~Hz}\right) ; 7.35-7.41$ (m, 4H, H6', H-6"', H-8', H-8'); 7.63-7.67 (m, 2H, H-7', H-7'”); 7.66 (d, 1H, H-5, J = 2.4 Hz); 7.78 (s, 1H, H-2); 7.96-8.07 (m, 3H, H-5', H-5"', H-7).

\subsection{Synthesis of 16-[5-(4-Iodophenyl)furan-2-yl]-1H,15H,16H- dibenzopyrano[3,4-g:4'3'-d] dioxocine-1,15-dione 11}

A mixture of compound $\mathbf{6 d}(0.4 \mathrm{mmol})$, dichloromethane $(0.8 \mathrm{mmol})$ and sodium hydroxide $(0.8 \mathrm{mmol})$ in toluene $\left(3 \mathrm{~cm}^{3}\right)$ was heated at $60^{\circ} \mathrm{C}$ for $117 \mathrm{~h}$. After cooling the solid was filtered off and crystallized in ethanol.

For $\mathrm{C}_{30} \mathrm{H}_{17} \mathrm{IO}_{7}$ (616.4): Mp: >350 ${ }^{\circ} \mathrm{C}$; yield: 47\%; ${ }^{1} \mathrm{H}$ NMR ( $\left.\mathrm{CF}_{3} \mathrm{COOD}\right): 4.36$ (s, $\left.2 \mathrm{H}, \mathrm{CH}_{2}\right) ; 5.32$ (s, $\left.1 \mathrm{H}, \mathrm{CH}\right) ; 6.57$ (d, $\left.1 \mathrm{H}, \mathrm{H}-3, \mathrm{~J}=3.3 \mathrm{~Hz}\right) ; 7.26-7.51$ (m, $9 \mathrm{H}, \mathrm{H}-4$, Ar - H); 7.64 - 7.70 (m, 2H, H -3',5'); 8.10 - 8.13 (m, 2H, H - 2'”, $12^{\prime \prime}$ ). 


\section{Results and discussion}

Reaction of 1 with 6-substituted 4-oxo-4H-chromene-3-carbaldehydes 2 led to bis(4-hydroxy-2-oxo- $2 \mathrm{H}$-chromen-2-ones 5 in 79-81\% yields after 3.5 h of heating. Microwave-assisted method gave the comparable yield of $\mathbf{5 a}$ (82\%), but the reaction time was remarkably shortened (4min). The use of 5-arylfuran-2-carbaldehydes 3 in reaction with 1 led to derivatives 6 in 50-68\% yields.. The effect of microwave irradiation was manifested in the remarkable shortening of reaction time from 51-93 $\mathrm{h}$ under classical heating to $1.5 \mathrm{~h}$ in microwave oven.

When 4-hydroxycoumarin $\mathbf{1}$ was treated with methyl 2-formyl-4H-furo[3,2b]pyrrole-5-carboxylate $\mathbf{4}$ in pyridine either by classical heating for 3 h or in microwave oven for $4 \mathrm{~min}$, product 7 was obtained in comparable yields (52\% and $56 \%$, respectively).

Epoxycoumarins 8 and $\mathbf{9}$ were obtained in $92 \%$ and $62 \%$ yields, respectively by the dehydratation of $\mathbf{5}$ or $\mathbf{6}$ in acetic acid / p-toluenesulfonic acid medium.

The heating of 4-hydroxycoumarin 1 and 6-bromo-4-oxo-4H-chromen-3carbaldehyde in acetic anhydride led to 3-acetyloxy derivative $\mathbf{1 0}$ in 66\% yield.

Dioxocine-1,15-dione 11 was prepared in $47 \%$ yield by reaction of $\mathbf{6 d}$ with dichloromethane and sodium hydroxide in toluene. The reaction required long reaction time $(117 \mathrm{~h})$. The pursuit to shorter the reaction time by the influence of microwave irradiation was not successful and only decomposition polymeric product was isolated. All structures were confirmed by ${ }^{1} \mathrm{H}$ NMR spectra.

\section{Conclusions}

4-Hydroxycoumarin 1 reacted with heterocyclic aldehydes 2-4 to give bisderivatives 5-7, which served as intermediates for synthesis of epoxycoumarins $\mathbf{8}, \mathbf{9}$, 2,4',4"'-triacethylderivative $\mathbf{1 0}$ and dioxocine-1,15-dione $\mathbf{1 1}$, respectively. Microwave irradiation has proved to be a suitable method for enhancement of reactions of 1 with aldehydes.

Acknowledgements: This work was supported by the Slovak Research Agency under the contracts No. VEGA 1/1005/09 and APVV-0006-07

\section{References}

GIOVANI, A., CRAVOTTO, G., TAGLIAPIETRA, S., FERRARO, S., NANO, G. M., PALISANO, G.: Synthesis of 3-Alkyl-4-hydroxycoumarins by Reductive Fragmentation of 3,3'-Alkylidene-4,4'-dihydroxybis[coumarins]. Helv. Chim. Acta, 74, 1991, 1451-1458.

HAMDI, N., PUERTA, M. C., VALERGA, P.: Synthesis, structure, antimicrobial and antioxidant investigations of dikumarol and related compounds. Eur. J. Med. Chem., 43, 2008, 2541-2548.

JUNG, J. CH., LEE, J. H., OH, S., LEED, J. G., PARKB, O. S.: Synthesis and antitumor activity of 4-hydroxycoumarin derivatives. Bioorg. Med. Chem. Lett., 14, 2004, 5527-5531. 
MANOLOV, I., MAICHLE-MOESSMER, C., DANCHEV, N.: Synthesis, structure, toxicological and pharmacological investigations of 4-hydroxycoumarin derivatives. Eur. J. Med. Chem., 41, 2006, 882-890.

MAO, P.CH.M, MOUSCADET, J. F., LEH, H., AUCLAIR, CH., HSU, L.Y.: Chemical modification of coumarin dimer and HIV-1 integrase inhibitory activity. Chem. Pharm. Bull., 50, 2002, 1634-1637.

STANCHEV, S., MOMEKOV, G., JENSEN, F., MANOLOV, I.: Synthesis, computational study and cytotoxic activity of new 4-hydroxycoumarin derivatives. Eur. J. Med. Chem., 20, 2007, 1-13.

YAMASHITA, J., TAKEDA, S., MATSUMOTO, H., UNEMI, N., YASUMOTO, M.: Studies on antitumor agents. VII. Antitumor activities of O-alkoxyalkyl derivatives of 2'-deoxy-5-trifluoromethyluridine. Chem. Pharm. Bull., 35, 1987, 2373-2381. 